\title{
Power Source for Wireless Sensors in Pipes
}

\author{
Sherif Keddis and Norbert Schwesinger \\ Microstructured Mechatronical Systems, Technical University of Munich, Munich D 80333, Germany
}

\begin{abstract}
In this paper, we present investigations on energy harvesters for wireless sensors inside pipes. The harvesters are of flexible piezoelectric PVDF (Poly-Vinylidene-Di-Fluoride) and aluminum-foils as electrodes. The layers were stacked alternating on each other and wound to a spool. An LDPE (low-density polyethylene)-film wraps the spool and prevents the inflow of liquids. A ring shaped bluff body was placed inside the pipe to induce turbulence in the fluid stream. As the harvesters have been arranged downstream of the bluff body, they were forced to oscillate independent of the media. This led to a polarization and a separation of electrical charges. Experiments were carried out in a wind channel as well as in a water pipe. In air, the spool oscillates with a frequency of about $30 \mathrm{~Hz}$, at a wind speed of about $7 \mathrm{~m} / \mathrm{s}$. A voltage of about $4 \mathrm{~V}$ (peak-peak) was measured. This delivers in case of impedance adjustment power values of about $0.54 \mu \mathrm{W}$. In water, oscillation starts at a speed above $0.6 \mathrm{~m} / \mathrm{s}$. The average oscillation frequency is about $18 \mathrm{~Hz}$. At a velocity of $0.74 \mathrm{~m} / \mathrm{s}$, a peak-peak-voltage up to about $2.3 \mathrm{~V}$ was found. In case of impedance adjustment, the power was about $0.33 \mu \mathrm{W}$. This power is stored in a capacitor. Assuming a data transmission unit consumes about $0.2 \mathrm{mWs}$ during one operational period of $1 \mathrm{~s}$, the duty cycle can be calculated to about $6.2 \mathrm{~min}$ for air harvesting and $10.1 \mathrm{~min}$ for harvesting in water.
\end{abstract}

Key words: Piezoelectric energy harvesting, pipe, PVDF-spool, bluff body, air, water.

\section{Introduction}

Energy harvesting was a scientific hype during recent years. Unfortunately, it is impossible to find a corresponding number of products and respectively companies in this field. A few SMA's (Small and Medium Enterprises) have been founded to develop, produce and sell appropriate energy harvesting devices. Until today, one can still find new foundations in this field. Nevertheless, many of these foundations are gone meanwhile. Although closing of enterprises is not an extraordinary event, the number of company closures at that early stage of development is quite astonishing. There exists obviously a contradiction between the demand of the market and the performance of the products. A major mistake is the ignorance of the performance of batteries as direct competitive products. Considering the scientific world, one can find countless research groups worldwide working in the energy harvesting field. Many of them received grands from governmental institutions or industrial funding

Corresponding author: Norbert Schwesinger, Dr.-Ing., research fields: energy harvesting, microfluidics and microactuators. organizations. The results of these groups are evident in confusing large numbers of papers in scientific journals as well as presentations at national or international scientific conferences. Several books on the topic complete the scope of scientific work [1-3]. It seems sometimes, that energy harvesting deals with itself and has been completely forgotten why it is needed. EH (Energy Harvesters) should serve as electrical energy supply for small consumers. They should usually convert energy losses of technical processes into electrical energy. Since this transducing requires often small physical effects, the conversion efficiency is usually very low. Since cost of EHs significantly exceeds that of batteries, the use of EHs must be technically and economically viable. In order to assess this properly, one can identify various criteria. Only if a foreseen application is able to fulfill one of the criteria, a use of EH is beneficial. If the application cannot fulfill any of the criteria, it is better to supply consumers with electrical energy by batteries or the power grid. The identified criteria are listed below [4]:

(1) No replacements of batteries during lifetime of the consumer possible; 
(2) Ability to deliver a constant amount of energy;

(3) Acting as EH and sensor simultaneously;

(4) Simultaneous use of one single conditioning circuit by numerous harvesting units;

(5) Ban on the use of power grids or batteries.

\section{Energy Harvesting Applications}

Flow monitoring systems offer an example of a beneficial application field for EHs. Sensors should be ideally placed inside the pipes to control flow parameters like velocity, density or viscosity but also chemical or biological sensors. Electric power grid or batteries can commonly supply these sensors with power. To connect the electric power grid with the sensors, pipes need to be opened for wiring. Unfortunately, this is one reason of leakage due to sealing complications. On the other hand, a replacement of depleted batteries is either complicated due to sealing problems or absolutely impossible when pipes are unreachable (e.g., buried). In all cases, the sensor connection is most accompanied by an economically unfavorable flow interruption. Circumstances like these completely fulfill the first criterion.

Arrangements of EH inside pipes have to supply the sensor but also the data transceiver with electrical power. Therefore, they have to generate a sufficient amount of energy in a limited time span. .

This paper describes results of several investigations conducted to determine the optimal configuration of piezoelectric EH in gas pipes as well as in water pipes. Goal was the generation of enough energy to power a wireless sensor and a transceiver unit in order to let them operate autonomously. First results show, that the use of PVDF (Poly-Vinylidene-Di-Fluoride)-foils is a very promising approach.

\section{Energy Harvesting in Flowing Media}

Several EHs which are able to generate electrical energy from flowing media have been presented previously. Typical concepts to achieve this are the piezoelectric conversion used [4-10]. Additionally, electromagnetic conversion was presented in Refs. [11, 12]. Electromagnetic harvesters loose not only efficiency with shrinking dimensions, they require a relative large movement of a magnet to a coil, or vice versa, too. Possible rotary concepts known from the macroscopic world, offer severe frictional losses and makes it difficult to scale them down (e.g., friction of rotational components) [13]. This and corresponding pressure drops make these EHs unsuitable for applications inside standard water pipes. Piezoelectric harvesters show a distinctly promising scalability. Last year's research results show a focusing towards at least two piezoelectric materials: PZT (lead-zirconate-titanate) [5, 6] and PVDF [4, 8, 9]. PVDF has much lower piezoelectric coefficients than PZT and let expect relatively low converted power. This makes PZT more attractive as active material. Unfortunately, PZT is brittle and shows fatigue during the operational life span. Additionally, placements of PZT in water could lead to the loss of all required electrical properties. PZT is a porous material which could possibly absorb water. Harvesting devices of PVDF can overcome all PZT-based problems, especially in water as flowing media.

\section{Design and Implementation}

The PVDF-harvesters are developed based on investigations in Refs. $[4,7,8,14]$. The basic idea is the creation of mechanical stress oscillations in piezoelectric materials by means of turbulence flow-induced in the wake of a bluff body. PVDF shows beside its excellent mechanical properties also a high chemical resistivity and no water absorption. This makes it well suited for in-pipe applications with no concern about aging, fatigue or other health issues. In order to overcome the low power output traced back to the lower piezoelectric coefficient, PVDF is shaped like a spool. First, two PVDF-films and two electrode layers of aluminum are alternately stacked and wound 
to a spool. Due to that and with the right orientation of the polarized PVDF-films, the active piezoelectric area is now doubled. With increasing number of windings, the active area increases linear. The PVDF-film used in this configuration has a width and thickness of $12 \mathrm{~mm}$ and $25 \mu \mathrm{m}$, respectively. The width of the aluminum-film is just $8 \mathrm{~mm}$, while the thickness is about $6.0 \mu \mathrm{m}$. The length of the layers is determined by the diameter of the mandrel and the number of windings. It can be approximated by using the volume $V$ of the foil in an unrolled form Eq. (1) and equating it with the volume of the wound foil on the mandrel (Eq. (2)):

$$
\begin{array}{r}
V=W \cdot L \cdot T \\
V=W \cdot \pi \cdot\left(r_{2}{ }^{2}-r_{1}{ }^{2}\right)
\end{array}
$$

where, $W$ denotes the width of the PVDF-film, $L$ its length and $T$ its thickness. The radius of the mandrel is given by $r_{1}$ while $r_{2}$ denotes the radius of the most outer winding, which is dependent on $n$, the number of windings:

$$
r_{2}=n \cdot T+r_{1}
$$

Inserting Eq. (3) in Eq. (2), and Eq. (2) in Eq. (1), and solving Eq. (1) for $L$ leads to:

$$
L=\frac{\pi\left[\left(n \cdot T+r_{1}\right)^{2}-r_{1}^{2}\right]}{T}
$$

For investigations in water as well as to seal this device against liquid penetration and in view of comparable results for both liquid and gaseous fluids, two layers of LDPE (low-density polyethylene) of $25.0 \mu \mathrm{m}$ thickness were added as the inner and outer winding of the spool. These two layers welded together form a sealed ring-shaped spool as energy harvester. LDPE was chosen as a material with a low Young's modulus allowing for an efficient energy transfer into the PVDF. The spool was fixed at the inner side of the pipe by a sophisticated fixation mechanism. In order to convert kinetic energy of the flowing media into mechanical oscillating stresses in the piezoelectric films, a ring-shaped bluff body was positioned upstream of the EH. This position ensures the flow of vortices generated at the bluff-body randomly across the spool surface. Since vortices present under pressure regions in their surrounding they force the spool to a movement in the direction of the vortex, thus generating stress in the layer and thereby electric charges. Since vortex shedding does not occur continuously from the bluff body but alternately, the vortices cause the harvester to oscillate.

\section{Experimental Investigations}

Tests were carried out in air as well as in water as flowing media. EHs were tested in a 1"-pipe using a closed-loop wind channel from "ELV" (Uniwind). Different EH configurations were tested depending on the wind speed of $7 \mathrm{~m} / \mathrm{s}$. The efficiency of the conversion of kinetic energy to mechanical stress inside the active layers was indicated by measuring the open-circuit voltage. Investigations in water show many unexpected difficulties. Main hurdle was the leak tightness of the harvester modules. Water often penetrates into the harvester and led to short circuits between the electrodes. Particular the connecting wires showed a high susceptibility for water penetration. Nevertheless, after countless faulty preparations, it was possible to achieve leak tight EHs. These devices were positioned in flow direction behind the ring shaped bluff-body. Corresponding to the knowledge from investigations in air the "reversed-eel" fixation mode was used preferably. Fig. 1 shows the experimental setup of investigations in water.

\subsection{Harvester Configuration}

It is known that most of the losses occur during the conversion of flow's kinetic energy into mechanical/vibrational energy [13]. Shape and fixation method of the EHs are therefore crucial. On basis of a mandrel diameter of $22 \mathrm{~mm}$, three separately tested fixation methods showed promising output signals. The first shape looks similar like a flag or "eel" $[8,14]$. One screw fixes the spool to the pipe bottom. The unfixed side can oscillate freely in the flow. Other 


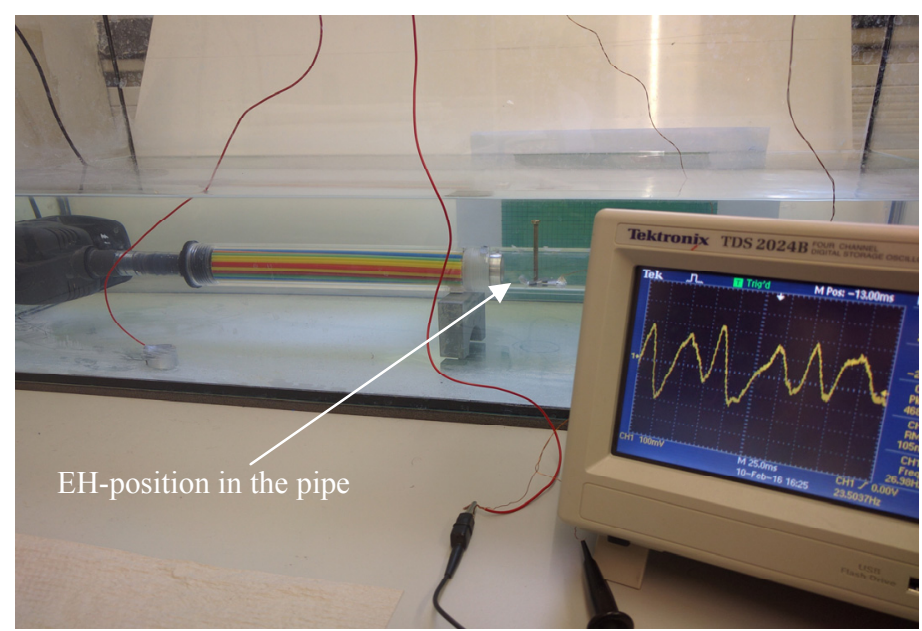

Fig. 1 Measurement setup for investigations in flowing water.

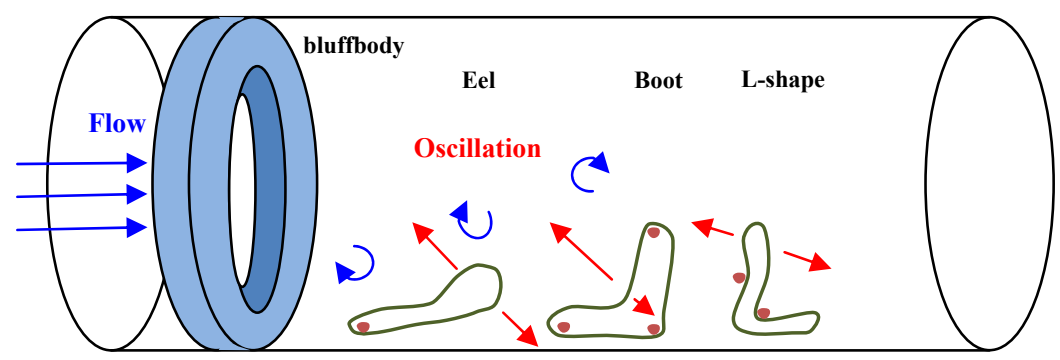

Fig. 2 Initial different fixing modifications investigated.

modifications of the fixing consist of more than 1 screw. Each modification has its own right in view of a high conversion capability.

Fig. 2 shows all different alternatives. The shape in the middle is fixed by three screws and looks like a boot. Vortices induced by the bluff body cause the side opposite to the flow direction of the harvester to bend outwards, while the pre-stress inside the materials try to return the layers to the starting position. The third fixing modification looks like an "L". This shape has a free horizontal oscillating end. Pre-stress inside the layers and the under pressure of the vortex cause the loop to hold up against the flow, while the screw fixed upstream of the harvester limits the backward movement.

In order to maximize the active area without exceeding a certain stiffness that could restrict the harvester's movement, the ideal number of windings had to be identified. Tests were made with two to six windings. Since four windings delivered best results in view of voltage output to material inset, the number of windings was kept to four windings. Best results were found with the reversed "eel"-shaped modification. Here, vortices can lift up the free end of the spool from the wall while internal spring forces try to hold it on the ground. Therefore, this modification was used throughout the remaining experiments to determine the optimal position of the harvester behind the bluff body. Fig. 3 shows the best fixing modification.

Depending on the distance between the bluff body and the fixation tool, the RMS (Root Mean Square)-voltage was measured. Regarding results are shown in Table 1.

As optimal distance between bluff-body and fixation point of the spool, a value of $2.5 \mathrm{~cm}$ was found for air (Table 1). Shorter or larger distances lead obviously to a drop down of the strain in the loop, hence to a drop of the RMS-voltage, too. The free end of the spool has a length of $13 \mathrm{~mm}$. 


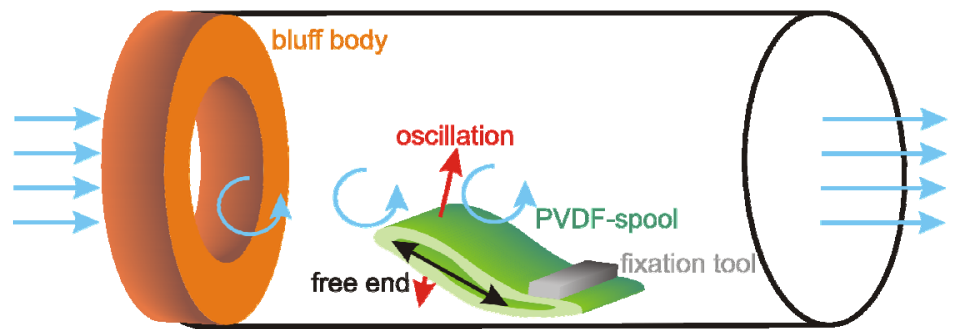

Fig. 3 "Reversed eel" fixing modification of the PVDF-spool.

Table 1 RMS-voltage of "reversed eel" at different positions from the bluff body in air.

\begin{tabular}{lllll}
\hline EH position & $2 \mathrm{~cm}$ & $2.5 \mathrm{~cm}$ & $3.5 \mathrm{~cm}$ & $5.5 \mathrm{~cm}$ \\
\hline RMS-voltage & $1.2 \mathrm{~V}$ & $1.4 \mathrm{~V}$ & $1.1 \mathrm{~V}$ & $0.8 \mathrm{~V}$ \\
\hline
\end{tabular}

Table 2 RMS-voltage of "reversed eel" for different numbers of windings in water.

\begin{tabular}{llll}
\hline No. of windings & 4 & 5 & 7 \\
\hline RMS-voltage & $0.5 \mathrm{~V}$ & $0.4 \mathrm{~V}$ & $0.1 \mathrm{~V}$ \\
\hline
\end{tabular}

Water shows a noticeably different behavior. Lower flow speed and higher density of the liquid led to changes in the optimal distance to the bluff body, the distance between bluff body and spool fixation point as well as the free end's length. It was impossible to test with free end's larger than $8 \mathrm{~mm}$ because the power of the vortices lifted the free end in a way that the continuous could and permanently bent the free end in flow direction preventing any oscillation. Spools with additional windings and a respectively higher stiffness should help to overcome this behavior. Even a shortening of the free end's length was another method to avoid the fold down of the spool. Although the distance between bluff body and spool fixation point has a big influence too, this value was constant at about $25 \mathrm{~mm}$ for all discussions in this paper. Table 2 shows results of the investigation made in flowing water.

Optimal impedance matching is the basis for the values of the RMS-voltage. The following sections describe results of the voltage output and the impedance matching.

\subsection{Voltage Output}

The influence of the flow velocity on the output energy is a basic interest. Measurements of the voltage and the oscillation frequency have been performed in air and in water. In both cases, the behavior is qualitatively very similar. At low flow speeds, the spool shows no movement. It lies on the bottom and seems to be unalterable by the flowing media. Only after exceeding a critical flow rate, the spool begins to oscillate. Nevertheless, the critical values of air and water differ by about one order. While the oscillation starts in water at about $0.6 \mathrm{~m} / \mathrm{s}$, the spool in air requires about $7 \mathrm{~m} / \mathrm{s}$ of flow speed. Although the free ends are not similar in air and water, this difference does not explain the distinction in the critical value. Much higher energy densities in water could possibly be the reason for this difference. The difference is shown in Fig. 4. From Fig. 4, one can conclude energy transfer in $\mathrm{EH}$-arrangements as described is only possible when the flow velocity exceeds a critical value. This value depends on the properties of the fluid but also on geometrical parameters. Assuming constant parameters of the spool, i.e., number of windings, width, length and free end, the size of the bluff body, the distance between the harvester and the bluff body as well as the orientation of the harvester in the flow direction can influence the energy transfer. At this early stage of investigation, it is impossible to report about these influencing parameters. Nevertheless, statements about this behavior will be given in next future.

\subsection{Impedance Matching}

After finding the best fixation and determining a good position inside the pipe that allows for obviously large mechanical strain, the harvesting output is characterized by means of the energy converted. Hereby, the energy supply of specific consumers is in focus in order to enable their operation for the entire 


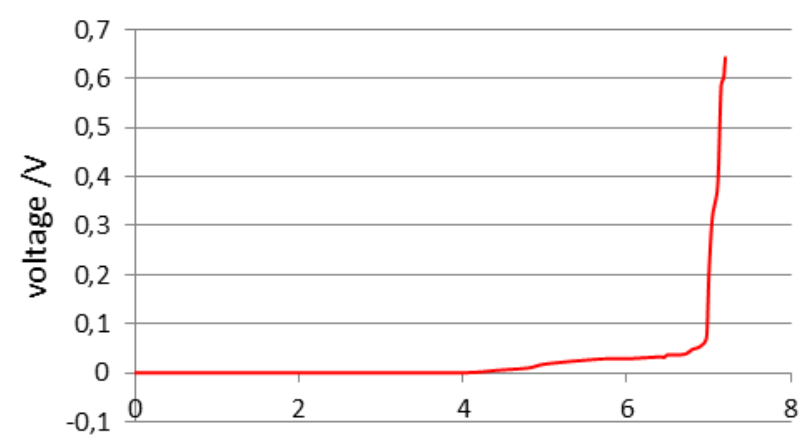

velocity $/ \mathrm{m} / \mathrm{s}$

(a)

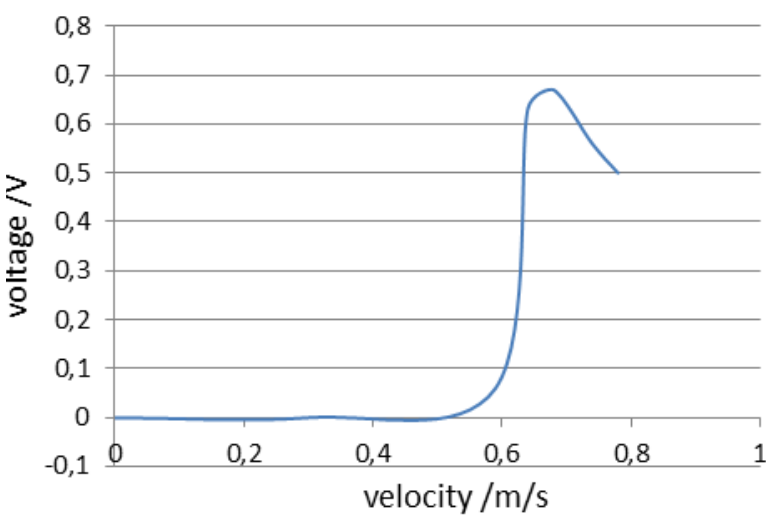

(b)

Fig. 4 RMS-voltage depending on the flow velocity: (a) air 4 windings; (b) water 5 windings.

life span under certain conditions. For example, if the load consists of a sensor and a data transfer unit, then the EH must provide both components with a sufficient amount of energy. Since parameter measuring and data transmission occur very often periodically, theses consumers need energy only during periodical operating times. Under these conditions, it is possible to define a so called "duty cycle". It can be different for various specific applications. For several data transmission operations, a duty cycle of about $20 \mathrm{~ms}$ is common whereby data measurement and transmission occur at 10 min each. During operation, the consumers require for instance $200 \mu \mathrm{Ws}$. Between two duty cycles, the EH must be able to generate this amount of energy.

The harvester delivers during operation a certain amount of energy. It can be measured across a load, which is in contact with the EH. Maximum energy transfer to the load is only possible, if the impedance of the generator and the electrical load is equal. In this case, an amount of exactly $50 \%$ of the energy generated is free for transfer. Goal of impedance matching is to find out the value of the load resistance which allows for a maximum energy transfer. Depending on the load resistance, voltage drop and current at the load resistance were measured. Power and energy were finally calculated by means of Ohm's law.

Fig. 5 shows the results of impedance matching for harvesting in air as well as in water.

For both harvesting methods, one can observe an increase of the RMS-voltage with increasing load resistance. Nevertheless, the oscillation frequencies of the harvesters differ due to the damping in the different media. In air, the frequency is in a range of about $30 \mathrm{~Hz}$. Water shows instead an average oscillation frequency of about $20 \mathrm{~Hz}$. The power shows for both media a typical maximum behavior. For the maximum value of the power, one can find a corresponding resistance. At this point, the impedance of the load equals the impedance of the EH. While the power peak of the harvester in air is very sharp, the maximum power shows in water a more rounded behavior. The height of the power differs unexpected only slightly. In air, the maximum power has a value of about $540 \mathrm{nW}$ at a resistance of about $3 \mathrm{M} \Omega$. EH in water shows a maximum power of $333 \mathrm{nW}$ at a resistance of about $480 \mathrm{k} \Omega$.

\section{Output Evaluation}

The measured voltage during the investigations indicates the efficiency of the conversion from kinetic flow energy of different fluids to mechanical strain inside the piezoelectric material. Considering the maximum power harvested in both media, one can state just a 1.6 times larger value in air instead of water. On the other hand, the flow rate of air is about 10.6 times larger than that of water. Obviously, the higher density of water has a much stronger effect on the energy conversion as the speed of the flow. Nevertheless, flow 


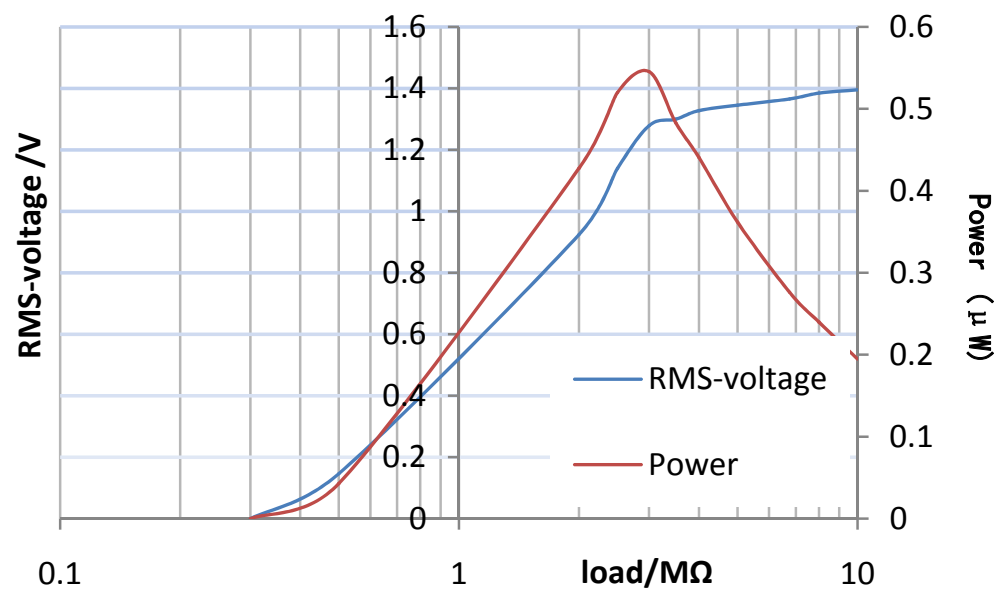

(a)

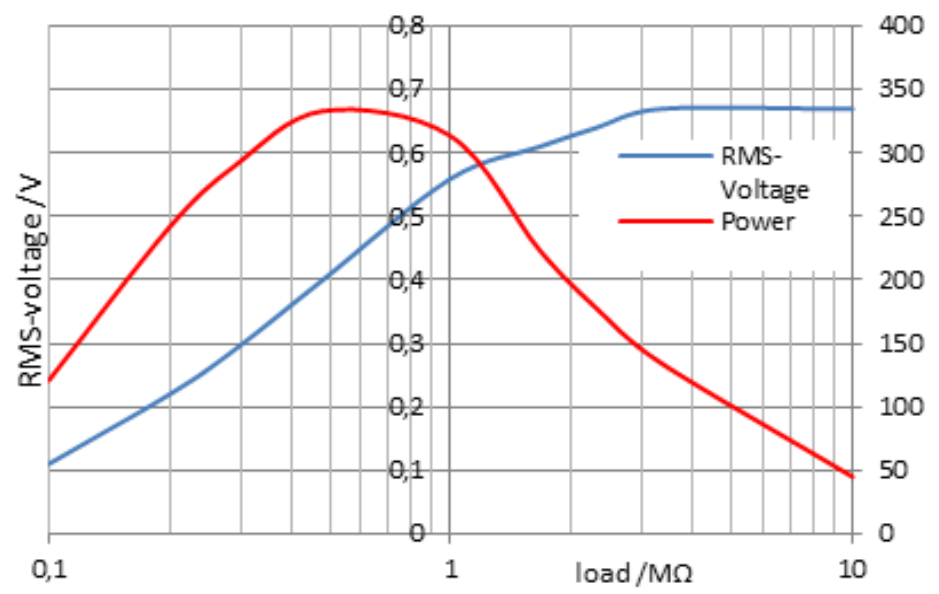

(b)

Fig. 5 Impedance matching of EH in flowing media: (a) air 4 windings; (b) water 5 windings.

velocities of water in the range of $7 \mathrm{~m} / \mathrm{s}$ are not usual and it makes no sense to operate with such high speeds in "normal" applications. Typical flow rates of drinking water are in the range of about $0.5 \mathrm{~m} / \mathrm{s} \sim 2 \mathrm{~m} / \mathrm{s}$, i.e., higher as during the measurements. Air flow velocities of $7 \mathrm{~m} / \mathrm{s}$ are not usual but possible in certain climate systems. Here, the average flow rate is about $3 \mathrm{~m} / \mathrm{s}$. Assuming a flow rate of $0.6 \mathrm{~m} / \mathrm{s}$ and a time of 10 min between 2 duty cycles, the water-EH can produce about $198 \mu \mathrm{Ws}$ of electrical energy. In air and a flow rate of $7 \mathrm{~m} / \mathrm{s}$, the $\mathrm{EH}$ can produce about $324 \mu \mathrm{Ws}$ during the same time. Taking into account a power consumption of about $200 \mu \mathrm{Ws}$ for a duty cycle of about $20 \mathrm{~ms}$, the $\mathrm{EH}$ in air is able to deliver this energy while the $\mathrm{EH}$ in water requires just a few seconds more between the duty cycles. However here unmatched flow rates were adopted. With other words, the harvester in air operates at the upper limit and the average energy converted decreases under normal conditions. For lower flow velocities, the design of the harvester must change in a way that the critical value at which the oscillation starts decreases. The harvester in water operates at the lower limit and the average energy converted energy increases under normal conditions. Due to this behavior, one can state that both energy harvesters are suited for applications in either real water pipes, gas pipelines or even in climate systems. They are able to supply a data transceiver and a sensor with a sufficient amount of energy as long as the time between duty cycles is well adapted. 


\section{Conclusions}

Improved flow EHs have been developed, which tackle the disadvantages of common known systems. The piezoelectric effect of PVDF converts energy from flowing media inside standard pipes into electrical energy. High flexibility and mechanical robustness of this material together with an outstanding bio-compatibility (lead-free) make PVDF a promising alternative to PZT used in most energy harvesters. Relatively low piezoelectric coefficients of PVDF are not unfortunate as long as it is possible to wind thin PVDF-films to a spool. This increases the active area and allows for a noticeable charge generation.

The conversion efficiency of the harvesters was tested in a wind channel and a water pipe. Tests in air have shown, the fixing modification of the spool inside the pipe is crucial for a high energy output. Different modifications were examined and most promising results were achieved using a "reversed-eel"-fixing modification. The spool faces the flow and oscillates due to vortices induced at a bluff body ring. Here it was found, that low flow velocities are not sufficient enough to produce vortices that are able to force the spool to oscillate. Only above a critical flow velocity the spools oscillate. The values of the critical flow velocities differ for water and air by about one order. Maximum power was measured under the condition of impedance matching. The EH continuously delivers a power of $0.54 \mu \mathrm{W}$ at air flow velocities above $7 \mathrm{~m} / \mathrm{s}$ and an adapted load of $3 \mathrm{M} \Omega$. This output power corresponds to an energy of $324 \mu \mathrm{Ws}$ in $10 \mathrm{~min}$, which is sufficient to supply a regarding sensor-transceiver-unit efficiently with an energy of $200 \mu \mathrm{Ws}$ during the duty cycle. EHs in water are able to continuously deliver a power of $0.33 \mu \mathrm{W}$ at water flow velocities above $0.65 \mathrm{~m} / \mathrm{s}$ and an adapted load of $480 \mathrm{k} \Omega$. This is sufficient for the power supply of a sensor-transceiver-unit with a power consumption of $200 \mu \mathrm{Ws}$ when the time between two duty cycles is larger than $10.1 \mathrm{~min}$.
Presently, the EHs are under examination in view of geometrical dependencies. It is expected that an optimal distance between the EH and the bluff body and an optimal size of the bluff body inflowing water, will lead to a noticeably higher power output. Even flow velocities above $0.65 \mathrm{~m} / \mathrm{s}$ could increase the converted electrical energy.

It could be shown, that in-pipe EH-applications will avoid usual sealing problems accompanied by penetrating pipes in order to connect the components and allow for a maintenance-free deployment of sensors at inaccessible locations.

\section{Acknowledgments}

This research was supported by the German Federal Ministry for Economic Affairs and Energy under the AiF sponsorship with the contract-No.: KF 3101002 UW3.

\section{References}

[1] Kazmierski, T. J., and Beeby, S. 2011. Energy Harvesting Systems. Berlin: Springer.

[2] Priya, S., and Inman, D. J. 2009. Energy Harvesting Technologies. Berlin: Springer.

[3] Tan, Y. K. 2012. Sustainable Energy Harvesting Technologies: Past, Present and Future. InTech. ISBN 978-953-307-438-2.

[4] Keddis, S., and Schwesinger, N. 2015. "Polymer Based Harvester as Power Source for Wireless Sensors in Pipes." Presented at ICAST: 26th International Conference on Adaptive Structures and Technologies, Blacksburg, VA.

[5] Kwon, S.-D. 2010. "A T-shaped Piezoelectric Cantilever for Fluid Energy Harvesting." Applied Physics Letters 97: 164102.

[6] Liu, H., Zhang, S., Kathiresan, R., Kobayashi, T., and Lee, C. 2012. "Development of Piezoelectric Microcantilever Flow Sensor with Wind-Driven Energy Harvesting Capability." Applied Physics Letters 100: 223905.

[7] Pobering, S., and Schwesinger, N. 2003. "A New Micro Hydro Power Device." In Proceedings of NSTI Nanotechnology Conference and Trade Show, 372-5.

[8] Taylor, G. W., Burns, J. R., Kammann, S. A., and Welsh, T. R. 2001. "The Energy Harvesting Eel: A Small Subsurface Ocean/River Power Generator." Oceanic Engineering 26: 539-47.

[9] Wang, D.-A., and Ko, H.-H. 2010. "Piezoelectric Energy Harvesting from Flow-Induced Vibration.” Journal of 
Micromechanics and Microengineering 20: 025019.

[10] Ye, G., and Soga, K. 2011. "Energy Harvesting from Water Distribution Systems." Journal of Energy Engineering 138 (1): 7-17.

[11] Hoffmann, D.,, Willmann, A., Göpfert, R., Becker, P., Folkmer, B., and Manoli, Y. 2013. :"Energy Harvesting from Fluid Flow in Water Pipelines for Smart Metering Applications." Journal of Physics: Conference Series 476: 012104.

[12] Torah, R., Glynne-Jones, P., Tudor, M., O’Donnell, T.,
Roy, S., and Beeby, S. 2008. "Self-Powered Autonomous Wireless Sensor Node Using Vibration Energy Harvesting." Measurement Science and Technology 19: 125202.

[13] Akaydin, H. D., Elvin, N., and Andreopoulos, Y. 2010. "Energy Harvesting from Highly Unsteady Fluid Flows Using Piezoelectric Materials." Journal of Intelligent Material Systems and Structures 21: 1263-78.

[14] Allen, J., and Smits, A. 2001. "Energy Harvesting Eel." Journal of Fluids and Structures 15: 629-40. 